\title{
Follicular thyroid carcinoma invades venous rather than lymphatic vessels
}

Xiaoqi Lin ${ }^{1 *}$, Bing Zhu', Yulin Liu², Jan F Silverman²

\begin{abstract}
Follicular thyroid carcinoma (FTC) tends to metastasize to remote organs rather than local lymph nodes. Separation of FTC from follicular thyroid adenoma (FTA) relies on detection of vascular and/or capsular invasion. We investigated which vascular markers, CD31, CD34 and D2-40 (lymphatic vessel marker), can best evaluate vascular invasion and why FTC tends to metastasize via blood stream to remote organs. Thirty two FTCs and 34 FTAs were retrieved for evaluation. The average age of patients with FTA was 8 years younger than FTC $(p=0.02)$. The female to male ratio for follicular neoplasm was 25:8. The average size of FTC was larger than FTA ( $p=0.003)$. Fourteen of 32 (44\%) FTCs showed venous invasion and none showed lymphatic invasion, with positive CD31 and CD34 staining and negative D2-40 staining of the involved vessels. The average number of involved vessels was $0.88 \pm 1.29$ with a range from 0 to 5, and the average diameter of involved vessels was $0.068 \pm 0.027 \mathrm{~mm}$. None of the 34 FTAs showed vascular invasion. CD31 staining demonstrated more specific staining of vascular endothelial cells than CD34, with less background staining. We recommended using CD31 rather than CD34 and/or D2-40 in confirming/excluding vascular invasion in difficult cases. All identified FTCs with vascular invasions showed involvement of venous channels, rather than lymphatic spaces, suggesting that FTCs prefer to metastasize via veins to distant organs, instead of lymphatic vessels to local lymph nodes, which correlates with previous clinical observations.
\end{abstract}

\section{Introduction}

Follicular thyroid carcinoma (FTC) accounts for 10 $17 \%$ of clinically evident thyroid malignancies [1-4]. It is more common in women, and tends to occur in patients in the fifth decade[1]. Survival is better in women and in patients younger than 40 years for male and 50 years for female [4-6]. Separation of FTC from follicular thyroid adenoma (FTA) is based on detection of vascular and/or capsular invasion[1]. The vascular invasion is almost never evident grossly[7]. Microscopically, the vessels should be located in or immediately outside the capsule (rather than within the tumor), and contain one or more clusters of tumor cells attached to the wall with protrusion into the lumen[1,7]. Often, the intravascular tumor foci are covered by endothelium, in a fashion similar to that of an ordinary thrombus[7]. The endothelial markers, such as CD31, factor VIII-related antigen, and Ulex europaeus, have been used in identifying vascular invasion [8-10]. When vascular invasion is

\footnotetext{
*Correspondence: xlin@northwestern.edu
'Department of Pathology, Northwestern Memorial Hospital, Northwestern

* Correspondence: xlin@northwestern.edu
'Department of Pathology, Northwestern Memorial Hospital, Northwestern University, Chicago, USA
}

( 2010 Lin et al; licensee BioMed Central Ltd. This is an Open Access article distributed under the terms of the Creative Commons

identified in FTCs, there is a prognostic significance based on the number of vessels involved $(<4$ or $\geq 4$ vascular invasion) [7,11-15].

Clinically, FTC tends to spread via blood stream, especially to the bones and lungs, and rarely to regional lymph nodes[1,16-20]. The skeletal metastases are usually multicentric but have a predilection for the shoulder girdle, sternum, skull, and iliac bone[21,22]. These metastases are common in the FTCs demonstrating extensive vascular invasion, but occur in fewer than $5 \%$ FTCs with minimal vascular invasion, and develop in less than $1 \%$ of the tumors diagnosed as carcinoma only on the basis of minimal capsular invasion $[14,23,24]$. Thirteen percentage of FTC smaller than $3 \mathrm{~cm}, 19 \%$ FTC between 3 to $6 \mathrm{~cm}$, and 33\% FTC > $6 \mathrm{~cm}$ show vascular invasion[25]. Up to $10 \%$ of patients with follicular or Hurthle cell carcinoma have tumors that aggressively invade structures in the neck or produce distant metastasis[26]. The metastases may exhibit a better differentiated appearance than the primary tumor, to the point of simulating normal thyroid as an expression of terminal differentiation (so-called "metastasizing 
adenoma", "malignant adenoma", or "metastasizing goiter")[7]. The majority, however, have poorly differentiated features, at least at the architectural level[20].

Occasionally, it can be challenging to detect vascular invasion on hematoxylin and eosin (H\&E) stained slides. Although vascular immunohistochemical (IHC) markers such as CD31[27,28], Factor VIII[9,10], Ulex europaeus [8] and CD34[27,29] have been used to identify vascular invasion in malignant neoplasms, the diagnostic value of these vascular markers compared to a specific lymphatic IHC marker, D2-40, in FTC has not been investigated $[30,31]$. In this study we investigated which vascular markers, CD31, CD34 and D2-40, can best identify vascular invasion in FTC, and studied whether venous or lymphatic vessels were involved. To date, no study has demonstrated a predilection of FTC for invading venous versus lymphatic vessels.

\section{Materials and methods \\ Selection of Cases}

The institutional review board of Allegheny General Hospital, Pittsburgh, PA approved the study. Thirty four follicular thyroid adenomas (FTA) and 32 follicular thyroid carcinomas (FTC) from 2000 to 2008 were retrieved from the hospital computer system. All FTCs were diagnosed when vascular and/or capsular invasion was histologically present. All patients were followed-up from 1 to 8 years, and none of the patients showed distal metastasis.

The average tumor size of FTA and FTC was $2.8 \pm 0.9$ $\mathrm{cm}$ with a range from 1.1 to $5.5 \mathrm{~cm}$ and $4.4 \pm 2.0$ with a range from 1.4 to $7.5 \mathrm{~cm}$, respectively $(\mathrm{P}=0.003)$. The average age of patients with FTA was approximately 8 years younger than those with FTC $(48.2 \pm 11.3$ vs. 56.7 \pm 11.6 years old, $\mathrm{P}=0.02$ ). The average age for male and female patients with FTC was $55.2 \pm 9.75$ and 56.2 \pm 13.0 , respectively $(\mathrm{P}=0.91)$. The female to male ratio with follicular thyroid neoplasm was $25: 8$, suggesting female predominance. The female to male ratios of FTA and FTC were 15:2 and 5:3, respectively $(\mathrm{p}=0.000007)$.

\section{Pathologic evaluation and immunohistochemistry}

Formalin-fixed (10 \% buffered formalin), routinely processed, hematoxylin and eosin-stained (H\&E) tissue sections including the entire capsule of the tumor were evaluated independently by 2 pathologists (XL and YL). Two blocks with capsule vascular invasion identified on H\&E stained slides or suspicious for vascular invasion from each case were chosen for immunohistochemical study.

Paraffin-embedded blocks were sectioned, deparaffinized, rehydrated, and blocked with methanolic 3\% hydrogen peroxide. Antigen retrieval was performed in citrate buffer ( $\mathrm{pH}$ 6.0). The immunohistochemical stains (IHC) for CD31 (catalog number CMA338, clone jc70a,
Mouse IgG1, no dilution, Cell Marquee, Hot Springs, AR), CD34 (catalog number 790-2927, clone QBEnd/10, mouse IgG1, no dilution, Ventana, Tucson, AR), and D2-40 (catalog number 730-01, clone D2-40, mouse IgG1, 1:50 dilution, Signet, Dedham, MA) on these slides were performed in an automated immunostainer with appropriate positive and negative controls. The detection was performed with Iview DAB detection kit (Catalog number 760-091, Vantana, Tucson, AZ). All slides were counterstained with hematoxylin and then were evaluated independently by 2 pathologists (XL and YL).

\section{Statistics}

The vascular invasion results from reviewing $H \& E$ and IHC stained slides by two pathologists were compared. Chi-square test and Student $\mathrm{T}$ test were used for statistical analysis.

\section{Results}

Identification of vascular invasion of follicular thyroid neoplasms

In this study, we identified vascular invasion in 13 FTC cases and no FTA cases by H\&E stained slides (Table 1 and Figure 1). IHC stain with CD31 and CD34 identified one more case in the FTC group, but no additional case in the FTA group. In the FTC group, one case was reported as "vascular invasion is equivocal", but capsular invasion was present. Reexamination of the equivocal case confirmed vascular invasion on repeat $H \& E$ slides, which further was confirmed with IHC stains for CD31 and CD34. These results suggested that IHC stain with CD31 and CD34 could help identify more vascular invasion and identify vascular invasion more accurately, and that IHC staining with CD31 or CD34 should be done especially if vascular invasion was equivocal. We also found that CD34 stained many non-endothelial cells, which occasionally compromised evaluation of vascular invasion. In contrast, CD31 was more specific than CD34 for vascular endothelial cells, making evaluation of vascular invasion much clearer.

The average number of vessels demonstrating vascular invasion was $0.88 \pm 1.29$ with a range from 0 to 5 . The average diameter of involved vessels was $0.068 \pm 0.027$ $\mathrm{mm}$.

The vessels invaded by FTC were venous rather than lymphatic

Antibodies against CD31[27,28] and CD34[27,29] are two widely-used markers of vascular endothelial cells and stain endothelial cells of both blood and lymphatic vessels, in contrast to D2-40 which stains only the endothelial cells of lymphatic vessels[30,31]. In this study, we found that all the vessels invaded by the FTCs were stained with CD31 and CD34 and none were stained with D2-40, supporting that venous rather than 
Table 1 IHC identification of vascular invasion of follicular thyroid neoplasms

\begin{tabular}{cccccc}
\hline Neoplasms & No. & H\&E & D2-40 & CD31 & CD34 \\
\hline Follicular Carcinoma & 32 & $41 \%(13 / 32)^{*}$ & $0 \%(0 / 32)$ & $44 \%(14 / 32)^{*}$ & $44 \%(14 / 32)^{*}$ \\
Follicular Adenoma & 34 & $0 \%(0 / 34)$ & $0 \%(0 / 34)$ & $0 \%(0 / 34)$ & $0 \%(0 / 34)$ \\
\hline
\end{tabular}

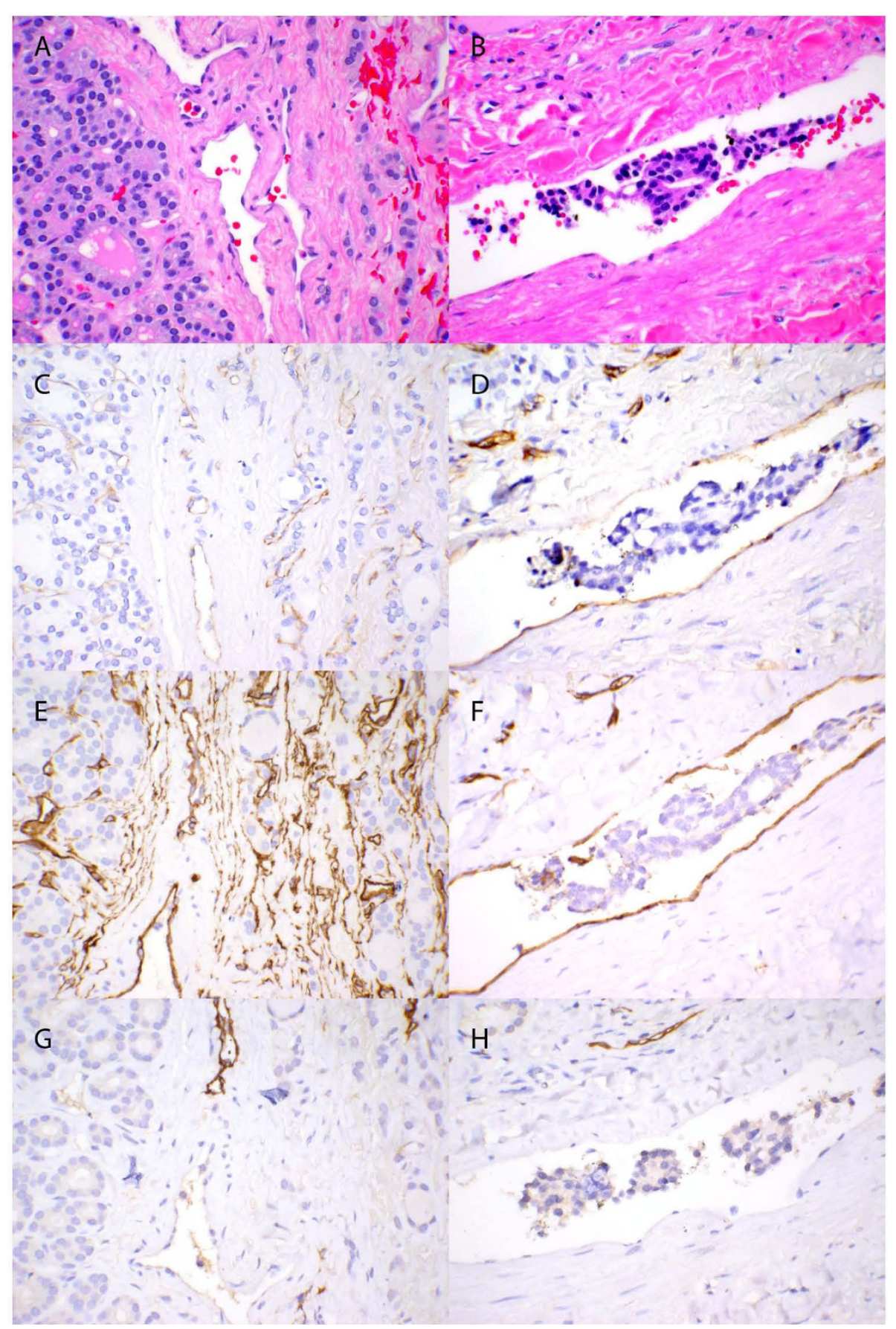

Figure 1 Follicular thyroid adenoma (A, C, E and G) and follicular thyroid carcinoma (B, D, F and H) (400x). Hematoxylin and eosin stains $(A$ and $B), C D 31$ stains (C and D), CD34 stains (E and F), and D2-40 stains ( $G$ and $H)$. 
lymphatic invasion was occurring (Table 1 and Figure 1 ). These results indicated FTC prefers to invade the venous rather than the lymphatic vessels.

\section{Discussion}

The diagnosis of FTC is contingent upon identifying capsular and/or vascular invasion[1]. Identification of the number of vessels involved by FTC is also important to predict prognosis $(<4$ or $\geq 4$ vascular invasion) $[7,11]$. In this study, we found that antibody against CD31 is better than antibodies against CD34 and D2-40 in identifying FTC vascular invasion and that FTC prefers to invade the venous rather than the lymphatic vessels.

Occasionally, it can be difficult to identify vascular invasion on $\mathrm{H} \& E$ stained slides[1,7]. One of our cases showed capsular invasion, but was initially equivocal for vascular invasion. However, vascular invasion was confirmed with IHC stains for CD31 and CD34. In addition, IHC stains for CD31 and CD34 identified vascular invasion in one case that was missed in the H\&E stained slides. Therefore, any suspicion for vascular invasion should be confirmed by IHC stains for CD31 and CD34, since IHC stains for CD31 and CD34 can more accurately and readily identify vascular invasion. We found that CD31 is preferable to CD34 for detecting vascular invasion of FTC due to less background staining. It is important to identify the vascular invasion, as the degree of vascular invasion by FTC has important prognostic implications[7,11].

Clinically, FTC is observed to metastasize hematogenously to distant organs in $7-40 \%$ of patients, with special preference to the lung and bones, rather than via lymphatics to regional nodes $[2,19,20,25,32-34]$. To date, no study has shown a preference of invading venous blood vessels versus lymphatic vessels by FTC. Antibodies against CD31 and CD34 stain both venous and lymphatic endothelial cells [27-29]. However, an antibody against D2-40 is a very sensitive and specific marker for lymphatic endothelial cells $[30,31]$. In this study, the endothelial cells of the vascular invasions in FTC were positive CD31 and CD34 and negative for D2-40, strongly suggesting that vascular invasion of FTC involves venous but not lymphatic vessels, which correlates with previous clinical observations $[19,20]$. However, the reason for this phenomenon is unknown, since papillary carcinoma is known to have a strong proclivity to involve lymphatic vessels with regional lymph node metastasis. In this study, we found that both D2-40 negative venous and D2-40 positive lymphatic vessels are present in the FTC and FTA capsules, excluding the possibility of lack of lymphatic vessels in the capsules. Future study on the preference of venous invasion by the FTC is important in order to further clarify the clinical behavior of FTC and to predict prognosis of FTC. In this study, no case showed distal and local metastasis during 1 to 8 years follow-up. Two cases with 4 or 5 venous vessels involved respectively also did not show distant metastasis 6 years after excision of FTC, but our observation may be limited by the relatively short follow-up. Another reason that may contribute to our finding is that with incoming use of thyroid fine needle biopsy to evaluate thyroid nodule in recent years, more FTC cases are potentially being diagnosed and treated at an earlier stage.

It was reported that FTC is more common in women, and tends to occur in patients in the fifth decade[1]. In this study, we had similar findings that the female to male ratio with follicular neoplasm was $25: 8$, with in 15:2 female to male ratio in FTA and 5:3 in FTC. These results not only suggested that follicular neoplasm especially FTA is much more common in female than male patients, but also male patients have higher risk than female patients to have FTC once they have thyroid follicular neoplasm.

In this study, we found that the average age of patients with FTA was about 8 years younger than those with FTC $(48.2 \pm 11.3$ vs. $56.7 \pm 11.6$ years old, $\mathrm{P}=0.02$, which was statistical significance). The average age of FTC patients is similar to a previous published report[1].

Four or more vessels involved by FTC has been proposed to have a worse prognosis[7,11-15]. Dr. Ghossein also has suggested to avoid the use of the term "minimally invasive" for those aggressive encapsulated follicular neoplasms with extensive angioinvasion, because the FTC with extensive vascular invasion implies a higher risk for recurrence[12]. It was suggested that the number of foci of vascular invasion should be mentioned in the pathology reports on FTCs, with a note regarding the increased risk of recurrence if at least 4 foci of vascular invasion are observed[12]. In our study, we had 2 cases with 4 and 5 vessels involved, respectively. Neither of the two patients show metastasis or local recurrence at 6 years after lobectomy and subtotal thyroidectomy. Tumor size greater than $4 \mathrm{~cm}$ has been shown to have worse prognosis[12]. In our study, we found that FTCs were on average significantly larger than FTA $(p=0.003)$.

In summary, we found that FTC invades venous vessels in the capsule rather than lymphatic vessels, which correlates with clinical observation that FTC more frequently metastasize via the blood stream to distant organs rather than regional lymph nodes. We recommended using IHC stain for CD31 rather than CD34 and D2-40 to confirm vascular invasion in difficult cases and predict distant metastasis and prognosis.

\section{Author details}

'Department of Pathology, Northwestern Memorial Hospital, Northwestern University, Chicago, USA. ${ }^{2}$ Department of Pathology, Allegheny General Hospital, Pittsburgh, USA. 


\section{Authors' contributions}

Dr. X Lin participated in its design, carried out the study, analyzed the data and drafted the manuscript. Dr. Y Lin participated in its design and analyzed the data. Drs. B Zhu and JF Silverman drafted the manuscript. All authors read and approved the final manuscript.

\section{Competing interests}

The authors declare that they have no competing interests.

Received: 26 August 2009

Accepted: 22 January 2010 Published: 22 January 2010

\section{References}

1. Sobrinho Simoes M, Asa SL, Kroll TG, Nikiforov Y, DeLellis R, Farid P, Kitamura Y, Noguchi SU, Eng C, Harach HR, et al: Follicular Carcinoma. World Health Organization Classification of Tumors: Pathology and Genetics of Tumors of Endocrine Organs Lyon: IARC PressDeLellis RA, Lloyd RV, Heitz PU, Eng C 2004, 67-72.

2. Gilliland FD, Hunt WC, Morris DM, Key CR: Prognostic factors for thyroid carcinoma. A population-based study of 15,698 cases from the Surveillance, Epidemiology and End Results (SEER) program 1973-1991. Cancer 1997, 79:564-573.

3. Crile G Jr, Pontius Kl, Hawk WA: Factors influencing the survival of patients with follicular carcinoma of the thyroid gland. Surg Gynecol Obstet 1985, 160:409-413

4. Samaan NA, Maheshwari YK, Nader S, Hill CS Jr, Schultz PN, Haynie TP, Hickey RC, Clark RL, Goepfert H, Ibanez ML, Litton CE: Impact of therapy for differentiated carcinoma of the thyroid: an analysis of 706 cases. $J$ Clin Endocrinol Metab 1983, 56:1131-1138.

5. Tubiana M, Schlumberger M, Rougier P, Laplanche A, Benhamou E, Gardet P, Caillou B, Travagli JP, Parmentier C: Long-term results and prognostic factors in patients with differentiated thyroid carcinoma. Cancer 1985, 55:794-804.

6. Cady B, Sedgwick CE, Meissner WA, Wool MS, Salzman FA, Werber J: Risk factor analysis in differentiated thyroid cancer. Cancer 1979, 43:810-820.

7. Rosai J: Surgical Pathology New York: Mosby, 92004.

8. Gonzalez-Campora R, Montero C, Martin-Lacave I, Galera H: Demonstration of vascular endothelium in thyroid carcinomas using Ulex europaeus I agglutinin. Histopathology 1986, 10:261-266.

9. Harach HR, Jasani B, Williams ED: Factor VIII as a marker of endothelial cells in follicular carcinoma of the thyroid. J Clin Pathol 1983, 36:1050-1054.

10. Stephenson TJ, Griffiths DW, Mills PM: Comparison of Ulex europaeus I lectin binding and factor VIII-related antigen as markers of vascular endothelium in follicular carcinoma of the thyroid. Histopathology 1986, 10:251-260.

11. Brennan MD, Bergstralh EJ, van Heerden JA, McConahey WM: Follicular thyroid cancer treated at the Mayo Clinic, 1946 through 1970: initial manifestations, pathologic findings, therapy, and outcome. Mayo Clin Proc 1991, 66:11-22.

12. Ghossein R: Problems and controversies in the histopathology of thyroid carcinomas of follicular cell origin. Arch Pathol Lab Med 2009, 133:683-691.

13. Collini P, Sampietro G, Pilotti S: Extensive vascular invasion is a marker of risk of relapse in encapsulated non-Hurthle cell follicular carcinoma of the thyroid gland: a clinicopathological study of 18 consecutive cases from a single institution with a 11-year median follow-up. Histopathology 2004, 44:35-39.

14. Lang W, Choritz H, Hundeshagen H: Risk factors in follicular thyroid carcinomas. A retrospective follow-up study covering a 14-year period with emphasis on morphological findings. Am J Surg Pathol 1986, 10:246-255.

15. Kahn NF, Perzin KH: Follicular carcinoma of the thyroid: an evaluation of the histologic criteria used for diagnosis. Pathol Annu 1983, 18(Pt 1):221-253.

16. Woolner LB, Beahrs OH, Black BM, Mc CW, Keating FR Jr: Classification and prognosis of thyroid carcinoma. A study of 885 cases observed in a thirty year period. Am J Surg 1961, 102:354-387.

17. Thompson NW, Nishiyama RH, Harness JK: Thyroid carcinoma: current controversies. Curr Probl Surg 1978, 15:1-67.
18. Young RL, Mazzaferri EL, Rahe AJ, Dorfman SG: Pure follicular thyroid carcinoma: impact of therapy in 214 patients. J Nucl Med 1980, 21:733-737.

19. Massin JP, Savoie JC, Garnier H, Guiraudon G, Leger FA, Bacourt F: Pulmonary metastases in differentiated thyroid carcinoma. Study of 58 cases with implications for the primary tumor treatment. Cancer 1984, 53:982-992.

20. Tickoo SK, Pittas AG, Adler M, Fazzari M, Larson SM, Robbins RJ, Rosai J: Bone metastases from thyroid carcinoma: a histopathologic study with clinical correlates. Arch Pathol Lab Med 2000, 124:1440-1447.

21. Nagamine Y, Suzuki J, Katakura R, Yoshimoto T, Matoba N, Takaya K: Skull metastasis of thyroid carcinoma. Study of 12 cases. J Neurosurg 1985, 63:526-531.

22. Pittas AG, Adler M, Fazzari M, Tickoo S, Rosai J, Larson SM, Robbins RJ: Bone metastases from thyroid carcinoma: clinical characteristics and prognostic variables in one hundred forty-six patients. Thyroid 2000, 10:261-268.

23. Cady B, Rossi R, Silverman M, Wool M: Further evidence of the validity of risk group definition in differentiated thyroid carcinoma. Surgery 1985, 98:1171-1178.

24. lida F: Surgical significance of capsule invasion of adenoma of the thyroid. Surg Gynecol Obstet 1977, 144:710-712.

25. Segal K, Arad A, Lubin E, Shpitzer T, Hadar T, Feinmesser R: Follicular carcinoma of the thyroid. Head Neck 1994, 16:533-538.

26. Sosa JA, Udelsman R: Total thyroidectomy for differentiated thyroid cancer. J Surg Oncol 2006, 94:701-707.

27. Tse LL, Chan I, Chan JK: Capsular intravascular endothelial hyperplasia: a peculiar form of vasoproliferative lesion associated with thyroid carcinoma. Histopathology 2001, 39:463-468

28. Niimi K, Yoshizawa M, Nakajima T, Saku T: Vascular invasion in squamous cell carcinomas of human oral mucosa. Oral Oncol 2001, 37:357-364.

29. Salizzoni M, Romagnoli R, Lupo F, David E, Mirabella S, Cerutti E, Ottobrelli A: Microscopic vascular invasion detected by anti-CD34 immunohistochemistry as a predictor of recurrence of hepatocellular carcinoma after liver transplantation. Transplantation 2003, 76:844-848.

30. Fogt F, Zimmerman RL, Ross HM, Daly T, Gausas RE: Identification of lymphatic vessels in malignant, adenomatous and normal colonic mucosa using the novel immunostain D2-40. Oncol Rep 2004, 11:47-50.

31. Kahn HJ, Marks A: A new monoclonal antibody, D2-40, for detection of lymphatic invasion in primary tumors. Lab Invest 2002, 82:1255-1257.

32. Dralle $\mathrm{H}, \mathrm{Gimm} \mathrm{O}$ : [Lymph node excision in thyroid carcinoma]. Chirurg 1996, 67:788-806.

33. Rao RS, Parikh HK, Deshmane VH, Parikh DM, Shrikhande SS, Havaldar R: Prognostic factors in follicular carcinoma of the thyroid: a study of 198 cases. Head Neck 1996, 18:118-124.

34. Mazzaferri EL, Jhiang SM: Long-term impact of initial surgical and medical therapy on papillary and follicular thyroid cancer. Am J Med 1994 97:418-428.

doi:10.1186/1746-1596-5-8

Cite this article as: Lin et al:: Follicular thyroid carcinoma invades venous rather than lymphatic vessels. Diagnostic Pathology 2010 5:8.

\section{Publish with Bio Med Central and every} scientist can read your work free of charge

"BioMed Central will be the most significant development for disseminating the results of biomedical research in our lifetime. "

Sir Paul Nurse, Cancer Research UK

Your research papers will be:

- available free of charge to the entire biomedical community

- peer reviewed and published immediately upon acceptance

- cited in PubMed and archived on PubMed Central

- yours - you keep the copyright 